\title{
Using an intense laser beam in interaction with muon/electron beam to probe the noncommutative QED
}

\author{
S. Tizchang, ${ }^{a}$ S. Batebi, ${ }^{a}$ M. Haghighat ${ }^{b}$ and R. Mohammadi ${ }^{c, 1}$ \\ ${ }^{a}$ Department of Physics, Isfahan University of Technology (IUT), \\ P.O. Box 84156-83111, Isfahan, Iran \\ ${ }^{b}$ Department of Physics, Shiraz University, \\ P.O. Box 71946-84795, Shiraz, Iran \\ ${ }^{c}$ Iran Science and Technology Museum (IRSTM), \\ P.O. Box 11369-14611, Tehran, Iran \\ E-mail: s.tizchang@ph.iut.ac.ir, s.batebi@ph.iut.ac.ir, \\ m.haghighat@shirazu.ac.ir, rmohammadi@ipm.ir
}

ABSTRACT: It is known that the linearly polarized photons can partly transform to circularly polarized ones via forward Compton scattering in a background such as the external magnetic field or noncommutative space time. Based on this fact we explore the effects of the NC-background on the scattering of a linearly polarized laser beam from an intense beam of charged leptons. We show that for a muon/electron beam flux $\bar{\varepsilon}_{\mu, e} \sim 10^{12} / 10^{10} \mathrm{TeV} \mathrm{cm}^{-2} \mathrm{sec}^{-1}$ and a linearly polarized laser beam with energy $k^{0} \sim 1 \mathrm{eV}$ and average power $\bar{P}_{\text {laser }} \simeq 10^{3} \mathrm{KW}$, the generation rate of circularly polarized photons is about $R_{V} \sim 10^{4} /$ sec for noncommutative energy scale $\Lambda_{\mathrm{NC}} \sim 10 \mathrm{TeV}$. This is fairly large and can grow for more intense beams in near future.

KEYWORDs: Non-Commutative Geometry, Effective field theories

ArXiv EPrint: 1608.01231

\footnotetext{
${ }^{1}$ Corresponding author.
} 


\section{Contents}

1 Introduction 1

2 Stokes parameters and Boltzmann equation 3

3 Time evolution of Stokes parameters vs. NC forward photon-fermion scattering

4 Charged lepton beams

5 Faraday conversion in Photon-Fermion beam interaction on NC space-time

6 Generation rate of circular polarization

7 Conclusion

\section{Introduction}

The Compton scattering is one of the most important scattering in physics particularly in the cosmology, astrophysics and astro-particle physics. Although this scattering can be the main source to generate a linear polarized wave, it cannot generate circular polarized wave. In general, the Stokes parameter $V$ which shows the amount of circular polarization of radiation is a pseudo-scalar quantity. Meanwhile, the Compton scattering of photons from unpolarized electrons is a parity invariant interaction. Consequently, for the primary photons without any circular polarization such a process can not lead to a nonzero circular polarization for the scattered photons because $V$ as a pseudo-scalar quantity cannot be formed from a combination of vectors and scalars [1] [also see [2-7]]. In fact, there is no many ways to generate circular polarization in a scattering process in the QED. Therefore, any experiment including the measurement of circular polarization can provide a way to understand more accurately the physics of scattering. Moreover, the linear polarization of a radiation may be converted to the circular polarization under the formalism of generalized Faraday rotation so-called Faraday conversion $[8,9]$. The evolution of the Stokes parameter $V$ in this mechanism can be obtained as [10]

$$
\dot{V}=2 U \frac{d \Delta \phi_{\mathrm{FC}}}{d t},
$$

where $\Delta \phi_{\mathrm{FC}}$ is the Faraday conversion phase shift and Stokes parameter $U$ indicates linear polarization. It is shown that in the presence of a background field such as Noncommutativity (NC) in space-time or external magnetic field, the generation of circular 
polarization by the Compton scattering is possible [11]. Meanwhile, it is recently shown that the photons of a linearly polarized laser beam can acquire circular or B-mode linear polarization by scattering off active neutrinos via a parity violation process. This phenomenon can possibly be used to understand the nature of neutrinos (Dirac or Majorana), to detect the fluxes of Cosmic Neutrino Background [12-14] and to gain some insight into the physics of active and light sterile neutrino oscillations [15]. Here we would like to consider NC-space as a nontrivial background to explore the polarization of photons in the Compton scattering. The NC field theory and its phenomenological aspects have been considered for many years without any sign in nature [17-26, 28, 29]. The NC space-time which is predicted in the string theory should have a scale $\Lambda_{\mathrm{NC}}$ of the order of the Planck scale. Nevertheless, it is believed that $\Lambda_{\mathrm{NC}}$ like the other new physics scales may has some impact at the $\mathrm{TeV}$ scale.

Constraints on noncommutative scale $\Lambda_{\mathrm{NC}}$ come from different experiments. For instance recently cosmological lower bound on noncommutative scale were derived by Planck data for $\mathrm{CMB} \Lambda_{\mathrm{NC}} \geq 20 \mathrm{TeV}$ [27]. The bound from Lamb shift in the hydrogen atom is in the range of $\Lambda_{\mathrm{NC}} \geq 0.1-1.5 \mathrm{TeV}[16,17]$. Besides, by comparing the Lorentz violating terms in the Higgs sector with the standard model extension the lower bound on noncommutative scale is obtained $\Lambda_{\mathrm{NC}} \geq 10^{6} \mathrm{TeV}$ [22]. To this end we consider those experiments which are based on the laser technologies. As in the Compton scattering in the NC-space the photons with the linear polarization transform partly to circularly polarized photons with a rate proportional to the NC-parameter, number of photons per pulse and the flux of fermions, one expects the enhancement of the rate of generation of the circular polarization with the intensities. Usually pulsed intense laser beams are designed for a wide-ranging experimental program in fundamental physics and advanced applications.

During the last 40 years, intensities of laser facilities have been increased from a few $\mathrm{KW} \mathrm{cm}{ }^{-2}$ to upper than $10^{22} \mathrm{~W} \mathrm{~cm}^{-2}$ such as the European Extreme Light Infrastructure (ELI) [30], the French Apollon laser [31], the Russian XCELS [32], the U.K. VULCAN laser [33] and the Astra-Gemini at RAL-CLF [34]. Besides the intensity, the average power of a laser is an important parameter. In recent years the leaser average powers are about a few watts which are higher than the range of tens of milliwatts to watts for the average powers of ruby lasers in the early 1960s. However, recently some progress has been made to improved the average power of ultrafast lasers [35], for instance see [36-39]. Furthermore, the Compton back scattering has been also considered since 1980s to produce photon beams. In this framework the Compton scattering of laser photons in the $\mathrm{eV}$ energy range with the high energy electrons in the range $E \sim \mathcal{O}(100 \mathrm{GeV})$, can produce a tight bunch of back scattered high energy photons [40-42]. Currently, there are gamma ray sources in the $\mathrm{MeV}$ range including machines at the LAL, the LUCX [43], ThomX [44] and the Compton backscattering at ATF [45].

In this work, we consider the forward Compton scattering through the collision of photons in a laser beam with an intense and high energy muon/electron beam in the noncommutative space to examine the generation of circular polarization. Consequently, we calculate the generation rate of the circularly polarized photons $R_{V}$ to explore the possibility of discovering the $\mathrm{NC}$ effects in different energy scales. It is clear to obtain a 
large value for $R_{V}$ to be measurable in lab, one needs intense muon/electron and laser beams. Therefore, an accelerator that can produce ultra-intense beams of muons and even electrons provides opportunities to discover the $\mathrm{NC}$ effect.

It should be noted that such intense lasers considering in this study can have nonlinear effects on the Compton scattering in the QED framework $[3,4]$ and consequently in the NCQED [5]. As it is already mentioned the unpolarized nonlinear Compton scattering in the ordinary QED cannot convert the linear polarization to the circular one due to its parity invariance. Nevertheless, the NCQED doesn't respect the parity symmetry therefore both the unpolarized linear and nonlinear Compton scattering through the NCQED can generate circular polarization from a linear one. But such intensities considered in this study for photon and fermion beams are low, so that nonlinear effects of NCQED can be safely neglected [5].

In section 2 we review the Stokes parameters and Boltzmann equation formalism. In section 3 the noncommutative standard model (NCSM) is briefly explained and the time evolution of the Stokes parameters related to the Compton scattering in NCSM is discussed. In section 4 we study the effect of space-time Noncommutativity on the collision of a laser beam with an ultra-intense beam of muon/electron. In section 5 we give an estimation on the generation rate of the circular polarization in such interactions. In section 6 some concluding remarks are given.

\section{Stokes parameters and Boltzmann equation}

Laser beam polarization can be characterized by four known Stokes parameters which are $I$ the total intensity of the beam, $Q$ and $U$ the linear polarization of the radiation and $V$ the difference between positive and negative circular polarization. Moreover, the density operator of an ensemble of photons in terms of the Stokes parameters is defined as follows

$$
\hat{\rho}=\frac{1}{\operatorname{tr}(\hat{\rho})} \int \frac{d^{3} \mathbf{k}}{(2 \pi)^{3}} \rho_{i j}(\mathbf{k}) D_{i j}(\mathbf{k}), \quad \rho=\frac{1}{2}\left(\begin{array}{cc}
I+Q & U-i V \\
U+i V & I-Q
\end{array}\right),
$$

where

$$
\begin{aligned}
I & =\rho_{11}+\rho_{22}, \\
Q & =\rho_{11}-\rho_{22}, \\
U & =\rho_{12}+\rho_{21}, \\
V & =i\left(\rho_{12}-\rho_{21}\right) .
\end{aligned}
$$

$\rho_{i j}(\mathbf{k})$ is the general density-matrix which is related to the photon number operator $D_{i j}^{0}(\mathbf{k})$ $\equiv a_{i}^{\dagger}(\mathbf{k}) a_{j}(\mathbf{k})$ where $a_{i}^{\dagger}(\mathbf{k})$ and $a_{j}(\mathbf{k})$ are photon creation and annihilation operators. The expectation value of the number operator is defined as

$$
\left\langle D_{i j}^{0}(\mathbf{k})\right\rangle \equiv \operatorname{tr}\left[\hat{\rho} D_{i j}^{0}(\mathbf{k})\right]=(2 \pi)^{3} \delta^{3}(0)\left(2 k^{0}\right) \rho_{i j}(\mathbf{k}) .
$$

The time evolution of the operator $D_{i j}^{0}(\mathbf{k})$, considered in the Heisenberg picture, is

$$
\frac{d}{d t} D_{i j}^{0}(\mathbf{k})=i\left[H, D_{i j}^{0}(\mathbf{k})\right]
$$


here $H$ is the full Hamiltonian. Meanwhile, the time evolution equation for the density matrix up to the first order of the interacting Hamiltonian $H_{I}^{0}(t)$ for photons can be written as

$$
(2 \pi)^{3} \delta^{3}(0)\left(2 k^{0}\right) \frac{d}{d t} \rho_{i j}(\mathbf{k})=i\left\langle\left[H_{I}^{0}(t), D_{i j}^{0}(\mathbf{k})\right]\right\rangle-\frac{1}{2} \int d t\left\langle\left[H_{I}^{0}(t),\left[H_{I}^{0}(0), D_{i j}^{0}(\mathbf{k})\right]\right]\right\rangle,
$$

where the first term on the right-hand side is a forward scattering term, and the second one is a higher order collision term, usual scattering cross section. The leading-order interacting Hamiltonian for photon-charged fermion is generally given by [46]

$H_{I}^{0}=\int d \mathbf{q} d \mathbf{q}^{\prime} d \mathbf{p} d \mathbf{p}^{\prime}(2 \pi)^{3} \delta^{3}\left(\mathbf{q}^{\prime}+\mathbf{p}^{\prime}-\mathbf{p}-\mathbf{q}\right) \times \exp \left[i t\left(q^{\prime 0}+p^{\prime 0}-q^{0}-p^{0}\right)\right]\left[b_{r^{\prime}}^{\dagger} a_{s^{\prime}}^{\dagger}(\mathcal{M}) a_{s} b_{r}\right]$,

where $\mathcal{M}$ is the amplitude of scattering matrix, $b_{r^{\prime}}^{\dagger}(\mathbf{k})$ and $b_{r}(\mathbf{k})$ are charged fermion creation and annihilation operators and $d \mathbf{q} \equiv \frac{d^{3} \mathbf{q}}{(2 \pi)^{3}} \frac{m_{f}}{q^{0}}$ and $d \mathbf{p} \equiv \frac{d^{3} \mathbf{p}}{(2 \pi)^{3} 2 p^{0}}$, with the same relations for $d \mathbf{q}^{\prime}$ and $d \mathbf{p}^{\prime}$, respectively.

The operator expectation values are given as a function of number density of fermions per unit volume, $n_{f}(\mathbf{q})$, where $\mathbf{q}$ denotes the momentum of fermions [46]. Then the energy density and pressure of fermions are defined as

$$
\varepsilon_{f}(\mathbf{x})=g_{f} \int \frac{d^{3} \mathbf{q}}{(2 \pi)^{3}} q^{0} n_{f}(\mathbf{x}, \mathbf{q}), \quad P_{f}=g_{f} \int \frac{d^{3} \mathbf{q}}{(2 \pi)^{3}} \frac{|\mathbf{q}|^{2}}{3 q^{0}} n_{f}(\mathbf{x}, \mathbf{q}),
$$

with $g_{f}$ shows the number of spin states or degrees of freedom.

\section{Time evolution of Stokes parameters vs. NC forward photon-fermion scattering}

In this section we explore the time evolution of the Stokes parameters via the forward photon-fermion scattering in the NC standard model. The noncommutative version of standard model is introduced by two different approaches. In the first approach which is based on the "Seiberg-Witten" maps, the symmetry group, number of gauge fields and particles are the same as the ordinary standard model [47]. Meanwhile, in the second approach the gauge group is $\mathrm{U}(3) \times \mathrm{U}(2) \times \mathrm{U}(1)$ which can be reduced to the standard model gauge group through appropriate symmetry breaking $[48,49]$. Here we follow the first approach to do our calculations.

In the both versions of the noncommutative standard model, the ordinary coordinates convert to the noncommutative operators with a canonical form as

$$
\left[\hat{x}^{\mu}, \hat{x}^{\nu}\right]=i \theta^{\mu \nu} \propto i \frac{1}{\Lambda_{\mathrm{NC}}^{2}},
$$

where $\theta^{\mu \nu}$ is the parameter of noncommutativity and $\Lambda_{\mathrm{NC}}=\frac{1}{\sqrt{\left|\theta^{\mu \nu}\right|}}$ is the noncommutative scale of energy. Also in the both version, Moyal-star product realization of the algebra are considered. The noncommutative parameter, $\theta^{\mu \nu}$, can be usually divided into two parts: 

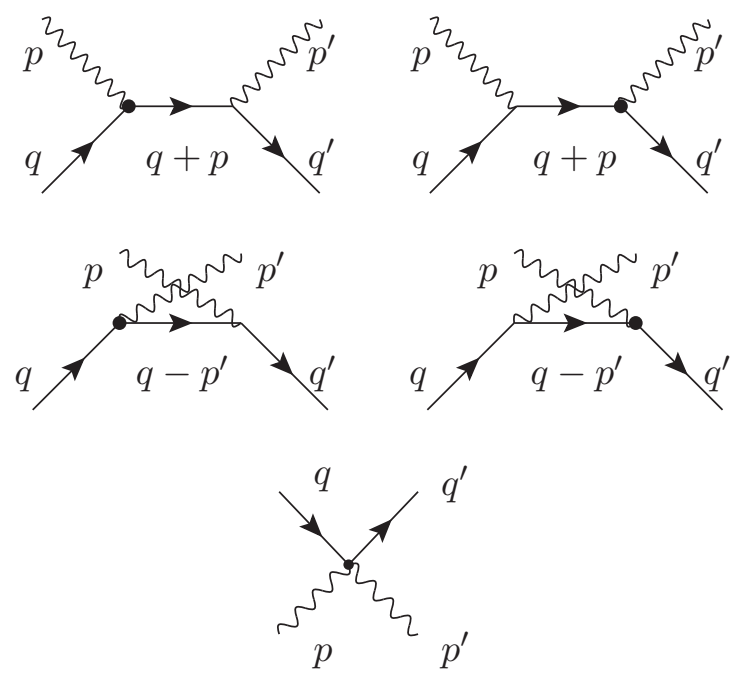

Figure 1. Compton scattering in NC standard model.

the time-space component $\theta^{0 i}$ and the space-space component $\theta^{i j}$ where $i, j, k=1,2,3$. The NC scale for the space-space and the time-space parts can be defined as [50]:

$$
\theta^{0 i}=\frac{1}{\Lambda_{\mathrm{TS}}^{2}} \hat{w}^{i}, \quad \theta^{i j}=\frac{1}{2 \Lambda_{\mathrm{SS}}^{2}} \varepsilon^{i j k} \hat{v}_{k},
$$

where $\hat{w}^{i}$ and $\hat{v}^{i}$ refer to fixed direction in space. Although the time-space component of the NC parameter has some problems with the unitarity, the quantum mechanics can become unitary in some cases for the time-like part of NC-parameter too [51, 52].

In the both approaches besides the corrections on the usual vertices new couplings also appear in comparison with the ordinary standard model.

Using the NCSM based on the "Seiberg-Witten" maps (first approach) [53], photon and charged fermion scattering can be described, up to the lowest order of NC-parameter, by the Feynman diagrams given in figure 1. In the QED part of the NCSM, the photonfermion vertex $f f \gamma$ up to the first order of $\theta$ is obtained as [53]

$$
\begin{aligned}
& \text { ie } Q_{f}\left[\gamma_{\mu}-\frac{i}{2} k^{\nu}\left(\theta_{\mu \nu \rho} p_{\text {in }}^{\rho}-\theta_{\mu \nu} m_{f}\right)\right]= \\
& \quad i e Q_{f} \gamma_{\mu}+\frac{1}{2} e Q_{f}\left[\left(p_{\text {out }} \cdot \theta \cdot p_{\text {in }}\right) \gamma_{\mu}-\left(p_{\text {out }} \theta\right)_{\mu}\left(\not \not p_{\text {in }}-m_{f}\right)-\left(\not p_{\text {out }}-m_{f}\right)\left(\theta p_{\text {in }}\right)_{\mu}\right],
\end{aligned}
$$

note that we define $A \cdot \theta \cdot B \equiv A_{\mu} \theta^{\mu \nu} B_{\nu}$, and also two photons can directly couple to two 
fermions in the NC space-time as follows [53]

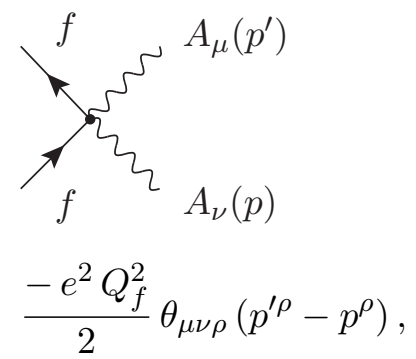

where photons momenta are taken to be incoming and $\theta^{\mu \nu \rho}$ is a totally antisymmetric quantity which is defined as

$$
\theta^{\mu \nu \rho}=\theta^{\mu \nu} \gamma^{\rho}+\theta^{\nu \rho} \gamma^{\mu}+\theta^{\rho \mu} \gamma^{\nu}
$$

Now we are ready to examine the time evolution of the Stokes parameters for photonfermion scattering in NCQED. It can be shown that the last diagram has not any contribution to the time derivative of circular polarization [54]. For the remaining diagrams given in figure 1 and using (2.9) one can find:

$$
\begin{aligned}
& i\left\langle\left[H_{I}^{0}(0), \mathcal{D}_{i j}(\mathbf{k})\right]\right\rangle=-\frac{e^{2} Q_{f}^{2}}{2}(2 \pi)^{3} \delta^{3}(0) \int d \mathbf{q} n_{f}(\mathbf{x}, \mathbf{q})\left(\delta_{i s} \rho_{s^{\prime} j}(\mathbf{k})-\delta_{j s^{\prime}} \rho_{i s}(\mathbf{k})\right) \\
& \times \bar{u}_{r}(q)\left\{\frac { 1 } { 4 k \cdot q } \left[q \cdot \theta \cdot \epsilon_{s^{\prime}}(k)\left(\not k\left(q+\not k+m_{f}\right) \notin_{s}(k)+\notin_{s}(k)\left(q-\not k+m_{f}\right) \not k\right)\right.\right. \\
& \left.+q \cdot \theta \cdot \epsilon_{s}(k)\left(\notin_{s^{\prime}}(k)\left(\not q+\not k+m_{f}\right) \not k+\not k\left(\not-\not k+m_{f}\right) \notin_{s^{\prime}}(k)\right)\right] \\
& \left.+\left[\epsilon_{s^{\prime}}(k) \cdot \theta \cdot \epsilon_{s}(k) \not k-k \cdot \theta \cdot \epsilon_{s}(k) \notin_{s^{\prime}}(k)+k \cdot \theta \cdot \epsilon_{s^{\prime}}(k) \notin_{s}(k)\right]\right\} u_{r}(q),
\end{aligned}
$$

and consequently one has

$$
\begin{aligned}
\frac{d}{d t} V(\mathbf{k})= & -\frac{i e^{2} Q_{f}^{2}}{4 m_{f} k^{0}} \int d \mathbf{q} n_{f}(\mathbf{x}, \mathbf{q})\left[\left(q \cdot \theta \cdot \epsilon_{1} q \cdot \epsilon_{1}-q \cdot \theta \cdot \epsilon_{2} q \cdot \epsilon_{2}\right) \mathrm{U}(\mathbf{k})\right. \\
& \left.-\left(q \cdot \theta \cdot \epsilon_{2} q \cdot \epsilon_{1}+q \cdot \theta \cdot \epsilon_{1} q \cdot \epsilon_{2}\right) Q(\mathbf{k})\right] .
\end{aligned}
$$

Using the matrix elements of the density operator and assuming $\theta^{0 i}=0$, the time variation of $V$ in $\mathrm{NC}$ space can be cast into:

$$
\dot{V}(\mathbf{k})=i \frac{3}{4} \frac{\sigma^{\mathrm{T}}}{\alpha k^{0}} \frac{m_{e}^{2}}{\Lambda_{\mathrm{NC}}^{2}} \frac{\bar{\varepsilon}_{f}}{g_{f}}(C Q+D U),
$$

where

$$
\begin{aligned}
& C=-\varepsilon^{i j k} \hat{q}_{i} \hat{v}_{k}\left(\epsilon_{1 j} \hat{q} \cdot \epsilon_{2}+\epsilon_{2 j} \hat{q} \cdot \epsilon_{1}\right), \\
& D=\varepsilon^{i j k} \hat{q}_{i} \hat{v}_{k}\left(\epsilon_{1 j} \hat{q} \cdot \epsilon_{1}-\epsilon_{2 j} \hat{q} \cdot \epsilon_{2}\right),
\end{aligned}
$$


and $\sqrt{2} \Lambda_{\mathrm{SS}} \sim \Lambda_{\mathrm{NC}}, \hat{q}=\frac{\vec{q}}{|q|}$ is the direction of fermion beam, $m_{e}$ is the mass of electron, $\sigma^{\mathrm{T}}$ is Thomson cross section, $\alpha=e^{2} / 4 \pi$ and $\bar{\varepsilon}_{f}$ is the energy density of fermion beam. One should note that the usual cross section for the Compton scattering in the NC-space $\sigma_{\mathrm{NC}} \sim \frac{\pi}{2} \alpha^{2} s / \Lambda^{4}[55]$ is too small in comparison with the order of magnitude of the forward scattering term which is obtained in (3.8) as $\kappa_{\mathrm{NC}}=\frac{3}{4} \frac{\sigma^{\mathrm{T}}}{\alpha g_{f}} \frac{m_{e}^{2}}{\Lambda_{\mathrm{NC}}^{2}}$. In fact for $s=\left(E_{1}^{2}+k_{0}\right)^{2} \sim$ $1 \mathrm{TeV}^{2}$ where $E_{1}$ is the energy of leptons and for $\Lambda_{\mathrm{NC}} \sim 1 \mathrm{TeV}$ one can easily find

$$
\kappa_{\mathrm{NC}} / \sigma_{\mathrm{NC}}=\frac{4}{\alpha} \frac{\Lambda_{\mathrm{NC}}^{2}}{s} \sim 10^{3},
$$

which shows $\sigma_{\mathrm{NC}}$ can be ignored in calculation for the Faraday conversion phase shift.

In the laboratory frame, we set the laser-photon momentum $\mathbf{k}$ in the $\hat{z}$-direction (the direction of incident laser beam), then the polarization vectors are defined as:

$$
\epsilon_{1}(k)=\hat{x}, \quad \epsilon_{2}(k)=\hat{y} .
$$

We may also consider the direction of muon beam, $\hat{q}$ in the $x-z$ plane, $\hat{q}=\sin \theta_{s} \hat{i}+\cos \theta_{s} \hat{k}$, then for the space-like component of noncommutative parameter one has

$$
\begin{aligned}
\Delta \phi & =\frac{\Delta V}{2 Q} \simeq \frac{3}{8} \frac{\sigma^{\mathrm{T}}}{\alpha k^{0}} \frac{m_{e}^{2}}{\Lambda_{\mathrm{NC}}^{2}} \frac{\bar{\varepsilon}_{f}(\mathbf{x}, \overline{\mathbf{q}})}{g_{f}} \Delta t\left(\frac{\sin 2 \theta_{s}}{2}-\sin ^{2} \theta_{s}\right) \hat{v} \cdot \hat{z} \\
& \approx 4.3 \times 10^{-36} \mathrm{~cm}^{2}\left(\frac{\mathrm{TeV}}{\Lambda_{\mathrm{NC}}}\right)^{2}\left(\frac{\bar{\varepsilon}_{f}(\mathbf{x}, \overline{\mathbf{q}})}{k^{0}}\right) \Delta t\left(\frac{\sin 2 \theta_{s}}{2}-\sin ^{2} \theta_{s}\right),
\end{aligned}
$$

where $\Delta t$ is the time interval of the laser beam interacting with the charged lepton beam.

\section{Charged lepton beams}

In the previous section we found how the circular polarization of photons depend on the beam parameters in the NC-space. Before calculating the Faraday conversion in fermionphoton scattering we briefly introduce the present available charged lepton beams. The charged particles suffering energy lost as the synchrotron radiation during the acceleration. However, for a particle with mass $m$ and beam energy $E$ in circular motion with radius $R$, the energy loss per revolution is given by $\Delta E \propto \frac{1}{R}\left(\frac{E}{m}\right)^{4}$ which is smaller for a larger radius or for a more massive particle. Therefore, accelerating the electron as the lightest charged particle to very high energy is a difficult task in the circular collider. In the LEP experiment, electrons were accelerated up to $E=100 \mathrm{GeV}$ [56]. Meanwhile, in a linear collider such as an International Linear Collider(ILC) one can reach to higher energies about $E=1 \mathrm{TeV}$ or even more.

Furthermore, in laser-plasma accelerators, electron can be accelerated to energies from hundreds of $\mathrm{MeV}$ [57-59] to multi-GeV energies [60, 61].

Muon as the 2nd lightest charged particle in nature but not stable, is almost 200 times more massive than the electron. In fact, the muon mean lifetime is very short about $\sim 2.2 \mu \mathrm{s}$, but enough to provide an intense beam which can be accelerated to high energy. However, pion decay can be usually used as a source for the muon production via $\pi^{+} \rightarrow$ 
$\mu^{+}+\nu_{\mu}$ and $\pi^{-} \rightarrow \mu^{-}+\bar{\nu}_{\mu}$. Meanwhile, colliding high energy protons with nuclei produce pions through interactions such as: $p+p \rightarrow p+n+\pi^{+}$and $p+n \rightarrow n+n+\pi^{+}$for the proton energies more than $400 \mathrm{MeV}$ and the double pion production in $p+p \rightarrow p+p+\pi^{+}+\pi^{-}$ at the larger energies. According to the momentum of the generated muon beam they are called "decay muon beam" or "surface muon beam". The first type of muon beam is about 80 percent polarized and have momentum from 40 to several hundreds of $\mathrm{MeV} / \mathrm{c}$. Such muon beams are available at PSI, TRIUMF, J-PARC ${ }^{1}$ and RIKEN-RAL. The second one is often known as surface or Arizona beam [62,63] that is 100 percent polarized and monochromatic and has kinetic energy $4.1 \mathrm{MeV}$. Such muon beams are available at PSI, ${ }^{2}$ TRIUMF, J-PARC, ISIS and RIKEN-RAL.

Besides, very low energy muon beams (ultra slow muons with energy at eV-KeV range) can be generated by reducing the energy of an Arizona beam [64] which is available in the PSI while the High-intensity low-energy muon beam is developing in J-PARC. Recently a multi-TeV Muon Collider, so called "Muon accelerator program" (MAP), has been planed. The purpose of this program is to develop the concepts and technologies required for the Muon Collider and Neutrino Factories [65, 66].

\section{Faraday conversion in Photon-Fermion beam interaction on $\mathrm{N}$ spa- ce-time}

For a typical muon beam with the number of muon per bunch $\sim 10^{12}$, the energy range $\bar{E}_{\mu} \approx|\overline{\mathbf{q}}| \sim 1 \mathrm{GeV}-1 \mathrm{TeV}$, the size of beam in the interaction region $\sim 1 \mathrm{~cm}$ and the bunch length about $\sim 1 \mathrm{~cm}$, one can estimate an average energy of flux per bunch as

$$
\bar{\varepsilon}_{\mu}(\mathbf{x}, \overline{\mathbf{q}}) \approx|\overline{\mathbf{q}}| n_{\mu}(\mathbf{x}, \overline{\mathbf{q}}) c \sim 10^{12} \mathrm{TeV} /\left(\mathrm{cm}^{2} \mathrm{~s}\right) .
$$

This type of muon beam has an angle divergence about $\theta_{\text {div }} \sim m_{\mu} / E_{\mu}$ which is negligible in the high energy muon beam accelerator which is the case for example in the MAP experiment $[65,66]$. To find the Faraday conversion for instance in Photon-Muon beams interaction we have for the interacting spot a spatial interval $\Delta d \sim 2 R_{0}+d \theta_{\text {div }} \sim 1 \mathrm{~cm}$ where $\mathrm{d}$ is the distance between muon beam and interaction spot with laser beam and a temporal interval of the order of $\Delta t \approx \Delta d / c \sim 10^{-10}$ section Therefore, for a laser beam with energy of photons about $k_{0} \sim 1 \mathrm{eV}$ the eq. (3.12) for the conversion $\left.\Delta \phi\right|_{\mu B}$ leads to

$$
\left.\Delta \phi\right|_{\mu B} \approx 10^{-22}\left(\frac{1 \mathrm{TeV}}{\Lambda_{\mathrm{NC}}}\right)^{2}\left(\frac{\bar{\varepsilon}_{\mu}}{10^{12} \mathrm{TeV} \mathrm{cm}^{-2} \mathrm{~s}^{-1}}\right)\left(\frac{\Delta t}{10^{-10} \mathrm{~s}}\right)\left(\frac{\mathrm{eV}}{k^{0}}\right) \sin ^{2} \theta_{s}
$$

where $\sin ^{2} \theta_{s} \sim 1$ when the laser beam is perpendicular to the muon beam. What is found in eq. (5.2) is the conversion for one interaction. However, the conversion can be enhanced by multiple interaction of the laser beam with muons which can be usually provided by a setup of suitable mirrors. If we suppose the mirrors have reasonable absorption coefficient about $C_{\mathrm{a}} \sim 10^{-5}$, the intensity of linearly polarized laser beam $Q$ reduces to $Q \rightarrow Q\left(1-C_{\mathrm{a}}\right)$

\footnotetext{
${ }^{1}$ Japan Proton Accelerator Research Complex.

${ }^{2}$ Swiss Muon Source $S \mu S$.
} 


\begin{tabular}{|cclr|}
\hline$E_{\mu}(\mathrm{TeV})$ & $\Lambda_{\mathrm{NC}}(\mathrm{TeV})$ & $k_{0}(\mathrm{eV})$ & $\sim \phi_{\mu B}$ \\
\hline 1 & 1 & 1 & $\sim 10^{-17}$ \\
& & 0.1 & $\sim 10^{-16}$ \\
\hline 1 & 20 & 1 & $\sim 10^{-19}$ \\
& & 0.1 & $\sim 10^{-21}$ \\
1 & 100 & 1 & $\sim 10^{-20}$ \\
\hline
\end{tabular}

Table 1. $\left.\Delta \phi\right|_{\mu B}$ for linearly polarized laser beam due to its interaction with muon beam for different values of $\Lambda_{\mathrm{NC}}$.

for each reflection. If we assume each laser pulse in its path can interact $N$-times with the muon beams, the maximum value of $N$ can be estimate as $\sum_{n=0}\left(1-C_{\mathrm{a}}\right)^{n} \approx \frac{1}{C_{\mathrm{a}}}[12]$. Therefore, eq. (5.2) for the $N$-times reflections leads to

$$
\left.\Delta \phi\right|_{\mu B} \approx 10^{-17}\left(\frac{1 \mathrm{TeV}}{\Lambda_{\mathrm{NC}}}\right)^{2}\left(\frac{\bar{\varepsilon}_{\mu}}{10^{12} \mathrm{TeV} \mathrm{cm}^{-2} \mathrm{~s}^{-1}}\right)\left(\frac{\Delta t}{10^{-10} \mathrm{~s}}\right)\left(\frac{\mathrm{eV}}{k^{0}}\right)\left(\frac{10^{-5}}{C_{\mathrm{a}}}\right) .
$$

The Faraday conversion phase shift of a linearly polarized laser beam due to its interaction with the muon beam for different values of $\Lambda_{\mathrm{NC}}$ is given in table 1. Meanwhile, one can use eq. (5.3) with appropriate changes for the other charged fermions. For instance, for the electron one can consider the appropriate parameters from the LEP experiment [56] in which the number of electrons per bunch is about $\sim 10^{11}$ locating at energies $\bar{E}_{e} \approx|\overline{\mathbf{q}}| \sim 100 \mathrm{GeV}$, where the size of beam in interaction region and bunch length are both about $\sim 1 \mathrm{~cm}$. Therefore, the mean energy of flux of the electron beam can be estimated as $\bar{\varepsilon}_{e} \sim 10^{10} \mathrm{TeV} \mathrm{cm}^{-2} \mathrm{~s}^{-1}$ which cast eq. (5.3) into

$$
\left.\Delta \phi\right|_{e B} \approx 10^{-19}\left(\frac{1 \mathrm{TeV}}{\Lambda_{\mathrm{NC}}}\right)^{2}\left(\frac{\bar{\varepsilon}_{e}}{10^{10} \mathrm{TeV} \mathrm{cm}^{-2} \mathrm{~s}^{-1}}\right)\left(\frac{\Delta t}{10^{-10} \mathrm{~s}}\right)\left(\frac{\mathrm{eV}}{k^{0}}\right)\left(\frac{10^{-5}}{C_{\mathrm{a}}}\right) .
$$

In this case the values of $\left.\Delta \phi\right|_{e B}$ for different values of $\Lambda_{\mathrm{NC}}$ in interaction of linearly polarized laser beam and the electron beam can be obtained which is given in table 2 .

\section{Generation rate of circular polarization}

In the previous section the Faraday conversion has been found to be at most $10^{-17}$ in the case of muons. Although this value seems to be small we show that can be large enough for the rate of generating circular polarization for the laser beam interacting with the muon/electron beam. To this end, we use eq. (3.12) in which $\Delta V(k)$ represents the number of generated circularly polarized photons with energy $|k|=k_{0} \sim 1 \mathrm{eV}$ per unit area $\left(\mathrm{cm}^{2}\right)$ per unit time $(\mathrm{s})$ for each laser pulse. The rate of the circular polarization generation for photons in the laser beam can be estimated as follows

$$
R_{V} \approx\left(\frac{\Delta V}{k^{0}}\right) \sigma_{\text {laser }} f_{\text {pulse }} \tau_{\text {pulse }}
$$




\begin{tabular}{|cclc|}
\hline$E_{e}(\mathrm{GeV})$ & $\Lambda_{\mathrm{NC}}(\mathrm{TeV})$ & $k_{0}(\mathrm{eV})$ & $\Delta \phi_{e B}$ \\
\hline 100 & 1 & 1 & $\sim 10^{-19}$ \\
& & 0.1 & $\sim 10^{-18}$ \\
\hline 100 & 20 & 1 & $\sim 10^{-21}$ \\
& & 0.1 & $\sim 10^{-20}$ \\
\hline 100 & 100 & 1 & $\sim 10^{-23}$ \\
& & 0.1 & $\sim 10^{-22}$ \\
\hline
\end{tabular}

Table 2. $\left.\Delta \phi\right|_{e B}$ for laser beam due to its interaction with electron beam different values of $\Lambda_{\mathrm{NC}}$.

where $\sigma_{\text {laser }}$ is the laser-beam size which is smaller than the charged lepton beam size $\Delta d$ and represents the effective area of photon-charged lepton interaction, $\tau_{\text {pulse }}$ is the time duration of a laser pulse and $f_{\text {pulse }}$ the laser repetition rate is the number of laser pulses per second. To have more efficiency, we assume that the laser and charged lepton beams are synchronized and the $f_{\text {pulse }}$ is equal to $f_{\text {bunch }}$ the repetition rate of beam which is the number of charged lepton bunches per second. Therefore, one can easily find the value of $R_{V}$ as follows

$$
R_{V} \simeq 10^{-16} f_{\text {pulse }} N_{\gamma}\left(\frac{1 \mathrm{TeV}}{\Lambda_{\mathrm{NC}}}\right)^{2}\left(\frac{\bar{\varepsilon}_{\mu}}{10^{12} \mathrm{TeV} \mathrm{cm}^{-2} \mathrm{~s}^{-1}}\right)\left(\frac{\Delta t}{10^{-10} \mathrm{~s}}\right)\left(\frac{\mathrm{eV}}{k^{0}}\right)\left(\frac{10^{-5}}{C_{\mathrm{a}}}\right),
$$

where $N_{\gamma}$ is the number of photons per pulse which can be obtained as

$$
N_{\gamma}=\frac{Q(k)}{k^{0}} \sigma_{\text {laser }} \tau_{\text {pulse }}=\frac{\varepsilon_{\text {pulse }}}{k^{0}},
$$

and the total energy of a laser pulse $\varepsilon_{\text {pulse }}=Q(k) \sigma_{\text {laser }} \tau_{\text {pulse }}$. Furthermore, the averaged power of a linearly polarized laser beam is approximately given by $\bar{P}_{\text {laser }}=f_{\text {pulse }} \varepsilon_{\text {pulse }}$ which cast eq. (6.2) for muon into

$$
\left.R_{V}\right|_{\mu B} \simeq 10^{-16} \frac{\bar{P}_{\text {laser }}}{k^{0}}\left(\frac{1 \mathrm{TeV}}{\Lambda_{\mathrm{NC}}}\right)^{2}\left(\frac{\bar{\varepsilon}_{\mu}}{10^{12} \mathrm{TeV} \mathrm{cm}^{-2} \mathrm{~s}^{-1}}\right)\left(\frac{\Delta t}{10^{-10} \mathrm{~s}}\right)\left(\frac{\mathrm{eV}}{k^{0}}\right)\left(\frac{10^{-5}}{C_{\mathrm{a}}}\right) .
$$

In eq. (6.4) we have only considered the forward scattering term. As is already mentioned the generation rate of the circular polarization $\left.R_{V}\right|_{\mu B} ^{\mathrm{NC}}$ due to the usual Compton scattering in NC-space $\sigma_{\mathrm{NC}} \sim \frac{\pi}{2} \alpha^{2} s / \Lambda^{4}[55]$ is very small and can be found as follows

$$
\begin{aligned}
\left.R_{V}\right|_{\mu B} ^{\mathrm{NC}} & <10^{-19} \frac{\bar{P}_{\text {laser }}}{k^{0}}\left(\frac{1 \mathrm{TeV}}{\Lambda_{\mathrm{NC}}}\right)^{2}\left(\frac{\bar{\varepsilon}_{\mu}}{10^{12} \mathrm{TeV} \mathrm{cm}^{-2} \mathrm{~s}^{-1}}\right)\left(\frac{\Delta t}{10^{-10} \mathrm{~s}}\right)\left(\frac{\mathrm{eV}}{k^{0}}\right)\left(\frac{10^{-5}}{C_{\mathrm{a}}}\right) \\
& \left.\ll R_{V}\right|_{\mu B},
\end{aligned}
$$

which shows its smallness and $\left.R_{V}\right|_{\mu B} ^{\mathrm{NC}}$ is negligible. Therefore, the main contribution on the generation rate from the $\mathrm{NC}$-space comes from (6.4). In fact, for a muon beam with $\bar{\varepsilon}_{\mu} \sim 10^{12} \mathrm{TeV} \mathrm{cm}^{-2} \mathrm{~s}^{-1}, \Lambda_{\mathrm{NC}}=1 \mathrm{TeV}$ and a linearly polarized laser beam of energy $k^{0} \sim$ 


\begin{tabular}{|cccl|}
\hline$E_{\mu}(\mathrm{TeV})$ & $\Lambda_{\mathrm{NC}}(\mathrm{TeV})$ & $R_{V}(1 / \mathrm{s})$ & $\bar{P}_{\text {laser }}(\mathrm{KW})$ \\
\hline 1 & 1 & $\sim 10^{4}$ & $\sim 10^{-2}$ \\
1 & 20 & $\sim 10^{4}$ & $\sim 10$ \\
1 & 100 & $\sim 10^{4}$ & $\sim 10^{2}$ \\
\hline
\end{tabular}

Table 3. The generation rate of circular polarization due to muon and laser beams interaction for $\Lambda_{\mathrm{NC}}=1 \mathrm{TeV}$ from atomic hydrogen $[16,17], \Lambda_{\mathrm{NC}}=20 \mathrm{TeV}$ obtained from Planck data for $\mathrm{CMB}[27]$ and $\Lambda_{\mathrm{NC}}=100 \mathrm{TeV}$.

\begin{tabular}{|ccll|}
\hline$E_{e}(\mathrm{GeV})$ & $\Lambda_{\mathrm{NC}}(\mathrm{TeV})$ & $R_{V}(1 / \mathrm{s})$ & $\bar{P}_{\text {laser }}(\mathrm{KW})$ \\
\hline 100 & 1 & $\sim 10^{4}$ & $\sim 1$ \\
100 & 20 & $\sim 10^{4}$ & $\sim 10^{3}$ \\
100 & 100 & $\sim 10^{4}$ & $\sim 10^{4}$ \\
\hline
\end{tabular}

Table 4. The generation rates of the circular polarization due to electron and laser beams interaction for $\Lambda_{\mathrm{NC}}=1 \mathrm{TeV}$ from atomic hydrogen $[16,17], \Lambda_{\mathrm{NC}}=20 \mathrm{TeV}$ obtained from Planck data for $\mathrm{CMB}[27]$ and $\Lambda_{\mathrm{NC}}=100 \mathrm{TeV}$.

$1 \mathrm{eV}$ and the average power $\bar{P}_{\text {laser }} \simeq 10 \mathrm{~W}$, the generation rate of the circularly polarized photons is about $\left.R_{V}\right|_{\mu B} \sim 10^{4} / \mathrm{s}\left(\sim 3 \times 10^{11}\right.$ year $)$. This rate seems to be large enough to be measured experimentally. The generation rates of the circular polarized photons $\left.R_{V}\right|_{\mu B}$ for different values of noncommutative scale are given in table 3 .

We can also give an estimation on the generation rate of the circular polarization due to the electron and laser beam interaction $\left.R_{V}\right|_{e B}$ as follows

$$
\left.R_{V}\right|_{e B} \simeq 10^{-18} \frac{\bar{P}_{\text {laser }}}{k^{0}}\left(\frac{1 \mathrm{TeV}}{\Lambda_{\mathrm{NC}}}\right)^{2}\left(\frac{\bar{\varepsilon}_{e}}{10^{10} \mathrm{TeV} \mathrm{cm}^{-2} \mathrm{~s}^{-1}}\right)\left(\frac{\Delta t}{10^{-10 \mathrm{~s}}}\right)\left(\frac{\mathrm{eV}}{k^{0}}\right)\left(\frac{10^{-5}}{C_{\mathrm{a}}}\right) .
$$

To obtain the rate $\left.R_{V}\right|_{e B} \simeq 10^{4} / \mathrm{sec}$ for an electron beam with $\bar{\varepsilon}_{e} \simeq 10^{10} \mathrm{TeV} \mathrm{cm}^{-2} \mathrm{~s}^{-1}$ and $\Lambda_{\mathrm{NC}}=1 \mathrm{TeV}$, a laser beam with average $\bar{P} \sim 1 \mathrm{KW}$ is needed which seems to be available in the near future with the present laser technologies [36, 38, 39]. Nevertheless, the generation rate of circular polarized photons due to the electron and laser beams interaction for different values of noncommutative scale and the laser average power is given in table 4.

\section{Conclusion}

We have considered the interaction of photons in an intense linearly polarized laser beam with ultra intense beams of muon and electron in the NC space time in the NCQED framework. Consequently, we have obtained the Faraday conversion phase shift $\Delta \phi$ and the generation rate $R_{V}$ of the circularly polarized photons in the interaction with muon/electron beam which are given in eqs. (5.3), (5.4) and eqs. (6.4), (6.6), respectively. We have discussed the possibility of using advanced laser and muon/electron beams to probe the noncommutative effects in such processes even for the $\mathrm{NC}$ scale as large as $10 \mathrm{TeV}$ with the 
available present technologies. In fact, for a muon/electron beam with a flux intensity of $\bar{\varepsilon}_{\mu, e} \sim 10^{12} / 10^{10} \mathrm{TeV} \mathrm{cm}^{-2} \mathrm{sec}^{-1}$ and a linearly polarized laser beam of energy $k^{0} \sim 1 \mathrm{eV}$ and an average power $\bar{P}_{\text {laser }} \simeq 10^{3} \mathrm{KW}$, the rate of generating of circularly polarized photons is about $R_{V} \sim 10^{4} / \mathrm{sec}$ for a noncommutative energy scale about $\Lambda_{\mathrm{NC}} \sim 10 \mathrm{TeV}$ [see tables 1, 2 for $\Delta \phi$ and tables 3,4 for $R_{V}$ ]. This rate seems to be large enough to be measured experimentally in near future.

Open Access. This article is distributed under the terms of the Creative Commons Attribution License (CC-BY 4.0), which permits any use, distribution and reproduction in any medium, provided the original author(s) and source are credited.

\section{References}

[1] V.B. Berestetskii, E.M. Lifshitz and L.P. Pitaevskii, Quantum Electrodynamics, Volume IV, Elsevier Ltd. (1980).

[2] A. Wightman, Note on Polarization Effects in Compton Scattering, Phys. Rev. 74 (1948) 1813.

[3] I.I. Goldman and V.A. Khoze, Polarization effects in Compton scattering by relativistic electrons, Soviet Phys. JETP 30 (1970) 501 [Zh. Eksp. Teor. Fiz. 57 (1969) 918] [InSPIRE].

[4] C. Harvey, T. Heinzl and A. Ilderton, Signatures of High-Intensity Compton Scattering, Phys. Rev. A 79 (2009) 063407 [arXiv:0903.4151] [InSPIRE].

[5] T. Heinzl, A. Ilderton and M. Marklund, Laser intensity effects in noncommutative QED, Phys. Rev. D 81 (2010) 051902 [arXiv:0909.0656] [INSPIRE].

[6] Z. Chang, H.-N. Lin and Y. Jiang, Gamma-ray burst polarization via Compton scattering process, Astrophys. J. 783 (2014) 30 [arXiv:1311.4313] [INSPIRE].

[7] V. Dinu, T. Heinzl, A. Ilderton, M. Marklund and G. Torgrimsson, Vacuum refractive indices and helicity flip in strong-field QED, Phys. Rev. D 89 (2014) 125003 [arXiv:1312.6419] [INSPIRE].

[8] M. Ruszkowski and M.C. Begelman, Circular polarization from stochastic synchrotron sources, Astrophys. J. 573 (2002) 485 [astro-ph/0112090] [INSPIRE].

[9] T.W. Jones and S.L. ODell, Transfer of polarized radiation in self-absorbed synchrontron source, Astrophys. J. 214 (1977) 522.

[10] A. Cooray, A. Melchiorri and J. Silk, Is the cosmic microwave background circularly polarized?, Phys. Lett. B 554 (2003) 1 [astro-ph/0205214] [INSPIRE].

[11] M. Zarei, E. Bavarsad, M. Haghighat, R. Mohammadi, I. Motie and Z. Rezaei, Generation of circular polarization of the CMB, Phys. Rev. D 81 (2010) 084035 [arXiv:0912.2993] [INSPIRE].

[12] R. Mohammadi and S.-S. Xue, Laser photons acquire circular polarization by interacting with a Dirac or Majorana neutrino beam, Phys. Lett. B 731 (2014) 272 [arXiv:1312.3862] [INSPIRE].

[13] R. Mohammadi, Evidence for cosmic neutrino background form CMB circular polarization, Eur. Phys. J. C 74 (2014) 3102 [arXiv:1312.2199] [InSPIRE]. 
[14] R. Mohammadi, J. Khodagholizadeh, M. Sadegh and S.-S. Xue, B-mode polarization of the CMB and the cosmic neutrino background, Phys. Rev. D 93 (2016) 125029 [arXiv: 1602.00237] [INSPIRE].

[15] P. Bakhti, R. Mohammadi and S.-S. Xue, Using strong intense lasers to probe sterile neutrinos, arXiv: 1403.7327 [INSPIRE].

[16] M. Chaichian, M.M. Sheikh-Jabbari and A. Tureanu, Hydrogen atom spectrum and the Lamb shift in noncommutative QED, Phys. Rev. Lett. 86 (2001) 2716 [hep-th/0010175] [INSPIRE].

[17] M. Haghighat and M. Khorsandi, Hydrogen and muonic-Hydrogen Atomic Spectra in Non-commutative Space-Time, Eur. Phys. J. C 75 (2015) 4 [arXiv:1410.0836] [InSPIRE].

[18] C.P. Martin and D.G. Navarro, The hybrid Seiberg-Witten map, its $\theta$-exact expansion and the antifield formalism, Phys. Rev. D 92 (2015) 065026 [arXiv: 1504.06168] [INSPIRE].

[19] R. Fresneda, D.M. Gitman and A.E. Shabad, Photon propagation in noncommutative QED with constant external field, Phys. Rev. D 91 (2015) 085005 [arXiv:1501. 04987] [INSPIRE].

[20] S. Batebi, M. Haghighat, S. Tizchang and H. Akafzade, Higgs Couplings in NonCommutative Standard Model, Int. J. Mod. Phys. A 30 (2015) 1550108 [arXiv:1410.7725] [inSPIRE].

[21] M. Ghasemkhani, R. Goldouzian, H. Khanpour, M. Khatiri Yanehsari and

M. Mohammadi-Najafabadi, Higgs production in $e^{-} e^{+}$collisions as a probe of noncommutativity, Prog. Theor. Exp. Phys. 2014 (2014) 081B01 [arXiv:1407.3167] [INSPIRE].

[22] S. Aghababaei, M. Haghighat and A. Kheirandish, Lorentz violation in the Higgs sector and the noncommutative standard model, Phys. Rev. D 87 (2013) 047703 [arXiv:1302.5023] [INSPIRE].

[23] A. Jafari, Photon dynamic in temporal noncommutative electrodynamics, Eur. Phys. J. C 73 (2013) 2271 [INSPIRE].

[24] N.G. Deshpande and S.K. Garg, Anomalous triple gauge boson couplings in $e^{-} e^{+} \rightarrow \gamma \gamma$ for noncommutative standard model, Phys. Lett. B 708 (2012) 150 [arXiv:1111.5173] [INSPIRE].

[25] R. Horvat, A. Ilakovac, P. Schupp, J. Trampetic and J. You, Neutrino propagation in noncommutative spacetimes, JHEP 04 (2012) 108 [arXiv:1111.4951] [INSPIRE].

[26] W. Wang, J.-H. Huang and Z.-M. Sheng, Bound on noncommutative standard model with hybrid gauge transformation via lepton flavor conserving $Z$ decay, Phys. Rev. D 88 (2013) 025031 [arXiv: 1306.1331] [INSPIRE].

[27] P.K. Joby, P. Chingangbam and S. Das, Constraint on noncommutative spacetime from PLANCK data, Phys. Rev. D 91 (2015) 083503 [arXiv:1412.6036] [INSPIRE].

[28] W. Wang, J.-H. Huang and Z.-M. Sheng, TeV Scale Phenomenology of $e^{+} e^{-} \rightarrow \mu^{+} \mu^{-}$ Scattering in the Noncommutative Standard Model with Hybrid Gauge Transformation, Phys. Rev. D 86 (2012) 025003 [arXiv: 1205. 0666] [INSPIRE].

[29] W. Wang, F. Tian and Z.-M. Sheng, Higgsstrahlung and pair production in $e^{+} e^{-}$collision in the noncommutative standard model, Phys. Rev. D 84 (2011) 045012 [arXiv:1105.0252] [INSPIRE].

[30] http://www.eli-beams.eu/science/lasers/.

[31] J.P. Zou et al, Design and current progress of the Apollon 10 PW project, High Power Laser Sci. Eng. 3 (2015) 1.

[32] http://www.xcels.iapras.ru/2013_news.html. 
[33] http://www.clf.stfc.ac.uk/Facilities/Vulcan/Vulcan+laser/12250.aspx.

[34] http://www.clf.stfc.ac.uk/Facilities/Astra/Astra+Gemini/12258.aspx.

[35] W.S. Brocklesby, Progress in high average power ultrafast lasers, Eur. Phys. J. Spec. Top. 224 (2015) 2529.

[36] W.S. Brocklesby et al., ICAN as a new laser paradigm for high energy, high average power femtosecond pulses, Eur. Phys. J. Spec. Top. 223 (2014) 1189 [INSPIRE].

[37] C. Teisset et al., $300 \mathrm{~W}$ Picosecond Thin-Disk Regenerative Amplifier at $10 \mathrm{kHz}$ Repetition Rate, in proceedings of the Advanced Solid-State Lasers Congress, Paris, France, 27 October-1 November 2013, G. Huber and P. Moulton eds., OSA Publishing, Washington, D.C. U.S.A. (2013), p. JTh5A.1.

[38] T. Mans, J. Dolkemeyer and C. Schnitzler, High Power Femtosecond Lasers, Laser Tech. J. 11 (2014) 40.

[39] J.P. Negel et al., $11 \mathrm{~kW}$ average output power from a thin-disk multipass amplifier for ultrashort laser pulses, Opt. Lett. 38 (2013) 5442.

[40] I.F. Ginzburg, G.L. Kotkin, V.G. Serbo and V.I. Telnov, Production of High-Energy Colliding $\gamma \gamma$ and $\gamma$ e Beams with a High Luminosity at Vlepp Accelerators, JETP Lett. 34 (1981) 491 [Pizma Zh. Eksp. Teor. Fiz. 34 (1981) 514] [InSPIRE].

[41] I.F. Ginzburg, G.L. Kotkin, V.G. Serbo and V.I. Telnov, Colliding $\gamma e$ and $\gamma \gamma$ beams based on the single-pass $e^{ \pm} e^{-}$colliders (VLEPP type), Nucl. Instrum. Meth. 205 (1983) 47 [INSPIRE].

[42] V.I. Telnov, Problems of multi-TeV photon colliders, Nucl. Instrum. Meth. A 472 (2001) 280 [hep-ex/0012047] [INSPIRE].

[43] M.K. Fukuda et al., Status and Future Plan of the Accelerator for Laser Undulator Compact $X$-ray Source (LUCX), in proceedings of the 1st International Particle Accelerator Conference (IPAC'10), Kyoto, Japan, 23-28 May 2010 [Conf. Proc. C 100523 (2010) TUPD089] [INSPIRE] and online pdf version at http://accelconf.web.cern.ch/AccelConf/IPAC10/papers/tupd089.pdf.

[44] A. Variola, The ThomX Project, in proceedings of the 2nd International Particle Accelerator Conference: IPAC 2011, San Sebastian, Spain, 4-9 September 2011 [Conf. Proc. C 110904 (2011) 1903] [INSPIRE] and online pdf version at http://accelconf.web.cern.ch/AccelConf/IPAC2011/papers/weoaa01.pdf.

[45] N. Delerue et al., High Flux Polarized Gamma Rays Production: First Measurements with a Four-mirror Cavity at the ATF, in proceedings of the 2nd International Particle Accelerator Conference: IPAC 2011, San Sebastian, Spain, 4-9 September 2011 [Conf. Proc. C 110904 (2011) 1446] [INSPIRE] and online pdf version at http://accelconf.web.cern.ch/AccelConf/IPAC2011/papers/tupo002.pdf.

[46] A. Kosowsky, Cosmic microwave background polarization, Annals Phys. 246 (1996) 49 [astro-ph/9501045] [INSPIRE].

[47] A.H. Chamseddine and A. Connes, Why the Standard Model, J. Geom. Phys. 58 (2008) 38 [arXiv:0706.3688] [INSPIRE].

[48] M. Chaichian, P. Prešnajder, M.M. Sheikh-Jabbari and A. Tureanu, Noncommutative standard model: Model building, Eur. Phys. J. C 29 (2003) 413 [hep-th/0107055] [INSPIRE].

[49] M. Chaichian, P. Prešnajder, M.M. Sheikh-Jabbari and A. Tureanu, Noncommutative gauge field theories: A No go theorem, Phys. Lett. B 526 (2002) 132 [hep-th/0107037] [INSPIRE]. 
[50] M. Haghighat, N. Okada and A. Stern, Location and Direction Dependent Effects in Collider Physics from Noncommutativity, Phys. Rev. D 82 (2010) 016007 [arXiv:1006.1009] [INSPIRE].

[51] A.P. Balachandran, T.R. Govindarajan, C. Molina and P. Teotonio-Sobrinho, Unitary quantum physics with time-space noncommutativity, JHEP 10 (2004) 072 [hep-th/0406125] [INSPIRE].

[52] A.P. Balachandran and A. Pinzul, On time-space noncommutativity for transition processes and noncommutative symmetries, Mod. Phys. Lett. A 20 (2005) 2023 [hep-th/0410199] [INSPIRE].

[53] B. Melic, K. Passek-Kumericki, J. Trampetic, P. Schupp and M. Wohlgenannt, The Standard model on non-commutative space-time: Electroweak currents and Higgs sector, Eur. Phys. J. C 42 (2005) 483 [hep-ph/0502249] [INSPIRE].

[54] S. Tizchang, S. Batebi, M. Haghighat and R. Mohammadi, Cosmic microwave background polarization in non-commutative space-time, Eur. Phys. J. C 76 (2016) 478 [arXiv: 1605.09045] [INSPIRE].

[55] S. Godfrey and M.A. Doncheski, Signals for noncommutative QED in e $\gamma$ and $\gamma \gamma$ collisions, Phys. Rev. D 65 (2002) 015005 [hep-ph/0108268] [INSPIRE].

[56] D. Brandt, H. Burkhardt, M. Lamont, S. Myers and J. Wenninger, Accelerator Physics at LEP, Rept. Prog. Phys. 63 (2000) 939 [CERN-SL-2000-037-DI] [INSPIRE].

[57] J. Faure et al., A laser-plasma accelerator producing monoenergetic electron beams, Nature 431 (2004) 541 [INSPIRE].

[58] C.G.R. Geddes et al., High-quality electron beams from a laser wakefield accelerator using plasma-channel guiding, Nature 431 (2004) 538 [INSPIRE].

[59] S.P.D. Mangles et al., Monoenergetic beams of relativistic electrons from intense laser-plasma interactions, Nature 431 (2004) 535 [INSPIRE].

[60] X. Wang et al., Quasi-monoenergetic laser-plasma acceleration of electrons to $2 \mathrm{GeV}$, Nat. Comm. 4 (2013) 1988.

[61] W.P. Leemans et al., Multi-GeV Electron Beams from Capillary-Discharge-Guided Subpetawatt Laser Pulses in the Self-Trapping Regime, Phys. Rev. Lett. 113 (2014) 245002 [INSPIRE].

[62] A.E. Pifer, T. Bowen and K.R. Kendall, A High Stopping Density $\mu^{+}$Beam, Nucl. Instrum. Meth. 135 (1976) 39 [INSPIRE].

[63] T. Bowen, The Surface Muon Beam, Phys. Today 38N7 (1985) 22 [inSPIRE].

[64] Y. Miyake et al., Ultra Slow Muon Microscopy for Nano-science, J. Phys. Conf. Ser. 302 (2011) 012038.

[65] US Muon Accelerator Program Report, Revised Completion Plan for the Muon Ionization Cooling Experiment (MICE) at Rutherford Appleton Laboratory, (2014) and online pdf version at https://web.fnal.gov/project/map/mice/CompletionPlan/DOE_Response_2014_ 0925_FINAL.pdf.

[66] J.-P. Delahaye et al., Enabling Intensity and Energy Frontier Science with a Muon Accelerator Facility in the U.S.: A White Paper Submitted to the 2013 U.S. Community Summer Study of the Division of Particles and Fields of the American Physical Society, arXiv:1308.0494 [FERMILAB-CONF-13-307] [INSPIRE]. 